\title{
Arriving at equivalence. Making a case for comparable general reference corpora in Translation Studies.
}

\section{Gill Philip}

\begin{abstract}
When multilingual corpora are used in translation studies, it is usually assumed that they are either translated (parallel) or comparable, or both; and that their size and text composition are analogous. As general reference corpora become more widely available, it is inevitable that these too should be used to compare and contrast SL norms, thus extending the definition of comparability to include text collections whose size and content may vary considerably, and which are nevertheless considered representative of their languages. This paper addresses the contribution of comparable reference corpora to the identification of translation equivalence. Focusing in particular on nativespeaker norms, it demonstrates how the effect of creative and idiosyncratic language can be identified and reproduced by the translator.
\end{abstract}

\section{Keywords}

General reference corpora, translation equivalence, synonymy, creativity

\section{Introduction}

In translation studies, multiple corpora are used to study like with like across languages. This may be achieved with translation corpora, in which the texts are the source language (SL) text in one corpus and translations of that text in the other(s); or, as is now more frequently the case, by using comparable corpora, composed of SL texts of a similar scope and content in each of the language(s) concerned. The adoption of comparable corpora has made it possible to move away from the study of translation as a product, and to focus instead on the identification, and reproduction in translated texts, of norms proper to the Target Language (TL) concerned. In other words, rather than studying previous translation choices (in a translation corpus), comparable corpora reveal how the word, phrase or term is actually rendered by native-speakers of the TL, allowing the translator to produce text which passes as native-like.

While small specialised corpora resolve issues pertinent to specialised languages or particular domains, it is beyond their scope to provide insights of a more general nature regarding the language as a whole. With the exception of technical language, which positively eschews turns of phrase, natural language abounds with idiomatic, metaphorical and other phraseological expressions, which create a range of difficulties for the translator. In particular, the peculiarity of phraseology in a SL text has to be accurately assessed: what meaning is being conveyed, and to what extend do the individual words convey that meaning? Is the expression conventional, is it novel, or is it a creative exploitation of a familiar expression? Is it marked or unmarked?

These problems, which contribute to the production of both oddities and normalisation in the TL, are matters which cannot be adequately addressed by small comparable corpora. However, translation corpora fare little better, as they offer previously-made translation choices, rather than provide the translator with a range of TL norms to choose from. Translation corpora and comparable corpora are restricted in size and scope, encapsulating single or closely-related genres, and are designed to address the specific needs of genre- or domain-specific translation, and they are 
neither large nor wide-ranging enough to be able to give an indication of more generalised norms within the languages under study. These norms contribute to the perception of naturalness in text, but are not as easily identified as terminological or structural aspects are.

It is here that the role of general reference corpora proves its worth. While terminology and other text- or genre-specific language are dealt with more profitably using relatively homogeneous comparable corpora, matters of a more wide-ranging and general nature can be usefully addressed by identifying and contrasting the language norms displayed in general reference corpora for the languages under study. This paper describes how comparable general reference corpora can be used to identify translation equivalents through the analysis and matching of cotextual patterns, and reveals how knowledge of these norms can be profitably exploited to avoid normalisation in the translation of creative and idiosyncratic language.

\section{Comparability and general reference corpora}

In extending the definition of comparable corpus to include text collections whose size and content may vary considerably, a number of matters must be addressed regarding the composition and size of the corpora, as well as their representativeness relative to their respective languages.[1]

In an ideal world, all general reference corpora would follow the same design criteria, making them of similar size, composed of similar text types in similar proportions. However in real life several standard models coexist, and each has its proponents and detractors. The Brown corpus (1 million words) and those modelled on it, including Frown, LOB and FLOB, comprises text samples of equal length, but as a corollary of this there are very few whole texts present in the data set, which means that organisational features may not be adequately represented. The British National Corpus (BNC) contains 100 million words of both spoken and written texts (10\% and $90 \%$ respectively) produced since 1964; sampling only takes place in texts which exceed 45,000 words in length, and is intended to avoid the risk of a single author's idiosyncrasies skewing the data.[2] In common with the Brown corpus, the BNC is static, and can only be kept up-to-date through re-issue. The corpus used in this study, the Bank of English, is a monitor corpus which undergoes constant updating and expansion, and now comprises 450 million words of running text.[3]

Publicly-accessible corpora for Italian are few and far between. This study draws on the Corpus di Italian Scritto (CORIS), which was the only Italian language corpus available at the time when this research was being carried out.[4] The composition of the 80 million-word CORIS is modelled on the written component of the Longman corpus of Spoken and Written English, making it qualitatively different from, as well as considerably smaller than, the Bank of English. Table 1 gives an indication of the distribution of text types in the two corpora.

Table 1. Proportions of text types in the Bank of English and CORIS

\begin{tabular}{|l|l|l|}
\hline text type & Bank of English & CORIS \\
\hline misc. journalism & $65.5 \%$ & $47.5 \%$ \\
\hline general prose & $17 \%$ & $25 \%$ \\
\hline academic prose & $1.5 \%$ & $12.5 \%$ \\
\hline legal prose & -- & $10 \%$ \\
\hline ephemera & $1 \%$ & $5 \%$ \\
\hline spoken & $15 \%$ & -- \\
\hline
\end{tabular}

Given these differences, it might appear far-fetched to describe the Bank of English and CORIS as comparable, but the most important consideration to bear in mind is that they are large general reference corpora, not small, text-or genre-specific comparable corpora. Dissimilar composition is 
not proof of incomparability: in accepting that languages are anisomorphic, it should come as no surprise that text types have different degrees of prominence and frequency of occurrence in different cultural and linguistic contexts, and that decisions regarding the representativeness of a general reference corpus for any language (or local language variety) must take this fact into account. In fact, the decision to model CORIS on LSWE was taken because its make-up was deemed more appropriate to Italian than the other contending models, most notably the BNC, LOB and Bank of English (Rossini Favretti 2000: 51). General reference corpora are expected to exemplify their languages in a balanced way, and as it is true that languages are not translations of each other, so representative samples of those languages need not mirror one other's composition. Viewed in this light, then, the comparability of the Bank of English and CORIS is not absolute, as two corpora constructed to the same design specification, but rather relative, with each corpus being independently constructed to take account of language-specific features and thus constitute a representative sample of the languages concerned.[5]

\section{Background to this research}

Having justified the use of the term comparable, it is now necessary to fill in some of the background to the applicability of comparable general reference corpora to the translation examples to be examined in 4.1.1 and 5.1.

The research from which this paper is drawn, Philip (2003), is a corpus-driven study of connotation in non-literary language. It examines the meaning of colour words as found in conventional linguistic expressions such as to see red, to feel blue, and green with envy, and explains what factors are responsible for activating the connotative meanings of the colour words when the expressions are used in running text. By comparing colour-word expressions with a number of near-synonyms which display similar phraseological patternings (e.g. to catch red handed, to catch in the act, to catch in flagrante delicto), it can be observed that the selection of one expression over another is largely predetermined by the situational context which the language is describing, and is both predicted and constrained by the regularity of patterning in the co-textual environment.

Although colour words are widely considered to be highly salient, Philip demonstrates that when they occur as part of conventional, non-compositional expressions, their meaning is subjected to the process of delexicalisation in the same way as any other component of a non-compositional chunk, in accordance with Sinclair's (1991) idiom principle and Louw's (2000) theory of progressive delexicalisation. As a result, the metaphorical-connotative meaning potential of these words remains latent, only rearing its head when the expressions undergo creative variation.

When the canonical forms of conventional expressions are altered, the way in which the phrase is interpreted changes radically, because the novel element has to be integrated into the whole. In order to do this, the non-compositional phrase is broken down into its component parts, and regains a degree of compositionality. The meaning is then reprocessed to make the relationship of the novel element to the underlying canonical form contingent; in doing so, meanings which are normally delexicalised regain a degree of saliency and metaphorical life. This can be observed by comparing the canonical forms in (1) and (2) with the creative variants in (3) and (4).

(1) The gang was finally caught red-handed in an armed police ambush in September 1992

(2) At the time it was claimed Kerr had been caught red-handed trying to smuggle arms to the Irish Republic.

(3) A car hi-fi thief was caught Simply Red-handed when he took a CD player into a store owned by his victim. 
(4) Mr. Green apparently had been caught scarlet-handed at his own blackmail game. Pictures of him with Miss Scarlet were found hidden in Scarlet's bedroom.

A similar phenomenon takes place when the cotext includes an element that favours a salient interpretation within the non-compositional phrase, as is the case with (4) which includes a colourword in the proper name, in addition to the colour word component of blackmail. The proximity of colour words in the phraseological core and in the co-text causes the delexical colour word to be relexicalised, thus re-activating the salient meaning.

In both these types of variation - phrase-internal, and phrase-external - the chunk is read as a phraseological palimpsest, the sum of the underlying conventional, delexicalised meaning and the novel, salient one that is superimposed on top of it.

\section{Identifying translation equivalents}

Conducting such a study with reference to two languages has translation as its ultimate aim. Monolingual reference corpora make it possible to identify the mechanisms which drive creativity in both languages concerned, and the results obtained demonstrate that the changes in meaning are governed by the same general principles - the combination of delexical meaning and a contextuallyrelevant salient add-on. This knowledge provides the basis for the informed translation of unconventional language, especially that found in literature, journalism and advertising, where word-play and anomalous language often falls victim to the normalisation process in translation (Kenny 2001: 65-69).

Translation involves a great deal of choice, whether explicit or implicit. Choice implies the selection of one interpretation, expressed by a particular sequence of words, over other possible contenders, with the aim to achieve as close an effect as possible to that obtained in the SL text. As Halliday puts it:

The translator is aware that a given item in the source has a set of possible equivalents in the target language. $[\mathrm{S} / \mathrm{he}$ is] aware that these are not free variants but they are contextually conditioned. By 'contextually conditioned' I do not mean that in a given context you must choose $\mathrm{A}$ and cannot choose $\mathrm{B}$ or $\mathrm{C}$, but that if you choose $\mathrm{A}$ or $\mathrm{B}$ or $\mathrm{C}$ then the meaning of that choice will differ according to what the context is. (Halliday 1992: 16)

So what is the translator's choice based on? Expert knowledge of the languages provides a substantial degree of intuition regarding equivalence; and language reference books and media fill in the gaps to a certain extent; but when the translator is faced with a range of apparently synonymous possibilities, how should he or she proceed? No two expressions are identical in meaning and function, but the fine details of the distinctions all too often escape our conscious knowledge.

In this case, the translation network comes into play. This need not involve the use of corpora, although both translation and comparable corpora clearly add detail which dictionaries and glossaries are not in a position to do. Reference to corpus data makes it possible to identify where differences and similarities lie across languages, thus fine-tuning the translator's knowledge. But while interesting as an academic exercise, the identification of exhaustive sets of equivalences involves umpteen passages of translation and back-translation. The example presented in Váradi and Kiss (2001), based on a translation corpus, demonstrates how cumbersome the procedure can be with new terms being added with each passage from one language to the other.[6] If the same procedure were carried out using comparable corpora (domain-specific or otherwise representing a 
restricted range of the languages concerned), the resulting translation web would be less messy and its realisation less onerous, if only because the language represented in the corpora is more homogeneous than that found in general reference corpora.

Whichever source of data is used, the building up of translation networks generally starts with a SL word form and a hypothetical translation in the TL (see Tognini Bonelli 2002: 81-2 for details of this procedure using two monolingual reference corpora, with the option of using a translation corpus, where available, to enrich the process). As the SL word form's patternings crystallise into functionally-defined "units of translation" (ibid. 80), each of these units must be matched up with a unit in the TL. However, the unit in the TL may be a leaky correspondence, either being too specific to cover all used of the SL unit, or, conversely, have a wider range of application than its SL equivalent. In the former case, further units in the TL have to be identified; in the latter, the distinct senses in TL unit have to be matched to new units in the SL. The network, or "translation web" (Tognini Bonelli 2001: 150-154), becomes more complex with every process of translation and back-translation as more and more terms are added and connected up with their equivalents in a potentially never-ending cycle.

\subsection{Translation equivalence and the paradigmatic axis}

One way to place a control on the network is to work on the basis that the SL word is one of several members of a larger semantic set, and as such it is distinguished and distinguishable from a range of near-synonyms. In this way, the analysis of the patternings of the SL term and its near-synonyms is carried out before embarking on the translation process. If the same procedure is applied to the posited TL equivalent term, i.e. that it is considered as a member of an analogous paradigm, for which the various patternings have to be identified, then the location of translation equivalents becomes a matter of matching up patternings, rather than searching for new expressions every time a new pattern appears. The correspondences are more detailed and accurate, and the tangled web of translations can be replaced by a more robust and linear schema of one-to-one correspondences that are arrived at independently of which language is to be considered the source or the target.

\subsubsection{Case study: go red}

The approach outlined above is best illustrated with a practical example. By taking the expression to go red as a point of departure, it is necessary to decide which other expressions belong to the paradigm in English, to posit an equivalent term in the TL (here, Italian), and to identify its nearsynonyms. This stage does not require use of a corpus, as the necessary information can be found in standard language reference works (mono-and bilingual dictionaries and thesauri). Thus, from the single item to go red, the English paradigm can be identified as to go red, to become red, to blush, to flush, to redden, and to turn red. The Italian equivalent selected is diventare rosso; the other members of its paradigm are arrossare, arrossarsi, arrossire, arrossirsi, farsi rosso (in viso/faccia), and far salire il sangue.[7]

The expressions are initially analysed without any reference being made to their translatability. The English terms are studied via corpus data from an English general reference corpus (in this case, the Bank of English), and the Italian terms are examined though Italian general reference corpus data (CORIS). Each term is broken down into its sense divisions (for example, separating out reflexive and non-reflexive forms of arrossar(si) and arrossir(si), and dividing the transitive and intransitive forms of flush) and extended units of meaning (Sinclair 1996). The expressions are profiled in terms of their collocational patterns, their colligational and semantic preferences, any extra-linguistic function or context of use that is indicated in the data, and, once the more detailed sub-senses and phraseologies have been identified, any apparent semantic prosody that these suggest is also noted.[8] Only once this detailed monolingual examination is complete is it possible to match up terms on the basis of the linguistic (and extra-linguistic) features that they have in common. This 
makes it possible to identify translation equivalence in a much more detailed and consistent way than any approach which takes the word alone as its starting point. By subdividing all the terms into their smaller units, it is possible to recognise, for example, that the presence of reflexivity can be a determining feature in arriving at translation equivalence (arrossare corresponds to redden, yet its corresponding reflexive form arrossarsi shares the same patterning as become red); or that the terms give rise to similar (and equivalent) phraseological or terminological constructions, as is the case with have the grace to blush and degnare di arrossire; and that the same subdivisions of meaning may be expressed in similar ways across both languages, for example go red as a beetroot and diventare rosso come un peperone which refer to embarrassment, while their related forms go red as a lobster and diventare rosso come un gambero describe sunburn.

\subsection{Manual and automatic profiling}

With its requirement for detailed analysis of members of a semantic set rather than of a single term, the paradigmatic model may give the impression of being perhaps unnecessarily time-consuming, but it should be remembered that it is proposed as an alternative to the existing - and considerably more onerous - method involving successive stages of translation and back-translation. If the intention is to compile some sort of translation database or to improve translators' reference works, then the corpus approach gives the most comprehensive account of how cotextual features contribute to the building up of meaning. It can provide extremely detailed information about how the words in question combine, the units of meaning that they generate, their textual positioning and their extra-linguistic function; all these aspects are potentially necessary to the translator.

Word profiling of the sort discussed here can be done manually, automatically or through a combination of both. The precise approach taken depends on time available, the potential of the analysis tools, and indeed the corpus itself, as some can only be interrogated through their built-in query software, which may limit the degree to which the analysis can be automated. The profiling discussed in this paper was carried out mainly by hand, the choice being determined by the corpora used: both the Bank of English and CORIS are only available by remote access, and can only be interrogated by their built-in query software.

Manual profiling is time-consuming, but generally highly accurate, as the human analyst is able to recognise semantic relations between collocates more easily than a computer can. Automatic profiling software is very sophisticated and detailed, but is limited in the extent to which it can cope with semantic relations. [9] To date, no applications can go beyond taxonomic semantic relations, i.e. hierarchical relations, lexical and semantic sets, to address the kind of ad hoc relations which humans create and interpret freely, which are based on shared attributes (see Glucksberg and Keysar 1993). When a regular pattern is found at the abstract level of semantic preference rather than in the concrete realm of countable, recurrent word-forms, the ability to appreciate such relations is especially important. Humans also find it easier to spot long-distance collocates (Seipmann, 2005), where the unit of meaning extends considerably farther than the extent of the concordance line on the computer screen; they are also able to make sense of incomplete text fragments or fractured phraseological patterns (Moon 1998) such as humorous exploitations of idiomatic expressions where the original is truncated or modified.

Manual profiling can of course be aided by corpus tools which guide the analyst towards particular patterns and phenomena. The "picture" option in the Bank of English's suite of tools (see Krishnamurthy 2000: 36-39) gives an overview of collocational frequency between $n-3$ and $n+3$ various positions around the search term, and was used extensively in the analysis of the English data in this study; most PC concordance packages now include very sophisticated tools for calculating collocations, patterns, n-grams and so on by frequency. As noted above, frequency 
counts and string-searches have their limitations, but they make initial profiling quick and reliable, with manual intervention confined to verification, fine-tuning and trouble-shooting.

\section{Native norms and creativity in translation}

In all attempts at pattern matching there will inevitably be some forms that appear not to have an equivalent, at least insofar as the paradigms studied are concerned. This is the case with the sense of turn red that collocates with leaves and berries. Although turn red can nearly always be translated as diventare rosso, this form in Italian never occurs with plant collocates to give the meaning "ripening", nor do any of its near-synonyms. In such a case as this, a new, related paradigm can be opened up for exploration (ripen and maturare, with their synonyms). On the other hand, should no translation be found to be appropriate, then, as Baker reminds us, "[a] certain amount of loss, addition, or skewing of meaning is often unavoidable" (1992: 57). The recurring phrase arrossire fino ai radici dei capelli (literally, "to blush to the roots of one's hair") is one such case in point. The translator should try to find an equivalent English expression (taking the verb as the base from), or use a paraphrase; in either case, the choice must combine the sense of blushing (including the semantic prosody), and the emphasis of extent: blush deeply, go bright red, turn beetroot. A quasiliteral rendition would be marked in English, and unless a particular effect was being sought, this would not be an appropriate translation solution for form which is unmarked in Italian. An untranslated borrowing would only be appropriate if attention were deliberately being drawn to the Italianness of the original. A literal translation with gloss might be appropriate in a commentary, but is unlikely to be so in narrative.

The adoption of a paradigm in translation adds a further degree of consciousness to the translation process. The translator is able to enter into an awareness of the language choices made by the author, and thus not only find the most accurate translation, but also note the differences between this term and the others which could have been used, but were not. This notion takes on particular importance when the language being translated differs from the norm - either in extreme cases such as the translation of poetry, or in the day-to-day inventiveness that characterises normal language use. Peculiarities and deviations from the SL norm can be assessed in relation to that norm and replicated in the TL, in full consciousness rather than by mere instinct. This means that the translation can match the effect of the original, because the mechanisms governing the effect can be identified and reproduced.

Using general reference corpora as an aid to the translation process means using data which makes it possible to assess and compare norms across the languages involved. Translated text does not fail utterly in this role, but it bears the sign of translation choices already made - for good or ill. With normalisation prevalent in translation of (apparently) atypical language, it is useful to be able to compare, as Kenny does (2001: 125ff.), translation corpus data with comparable corpus data, and to do so both for the SL and the TL.

\subsection{Expressing emotion through colour}

Colour words are typically used in European languages to express emotional states, mainly because there is a fairly transparent metonymical connection between, for instance, adrenaline speeding up the flow of blood through the body, and the face becoming flushed or red. So it is justifiable to expect that colour-word expressions should be used to refer to the manifestation of emotion in several languages. What may come as something of a surprise, however, is that the colour words typically used are not necessarily the same. For example, within Europe, English is odd in that it associates the colour green with envy, when other languages prefer yellow, the colour of bile; and 
blue meaning depressed (yet grey depressing) is far from universal. But the non-equivalences do not end here.

Casting aside any cultural reasons why colours and emotional states should not correspond exactly (see Nieimeier 1998; Philip 2003: 151-164), the fact remains that there is a degree of language variation in this area, and that a translator should be in a position to address it appropriately. Consider the following corpus extracts (5-7), in which rabbia (rage) is assigned different colours nero (black), viola (purple), and verde (green).

(5) Chi sta vicino al Castel de' Britti, dice che è nero di rabbia, che sogna la rivincita.

(6) È viola di rabbia, una furia scatenata

(7) Quando mia nonna le ha risposto: "Speriamo di no, altrimenti verrà fuori una puttana come te!", ho visto mia madre diventare verde di rabbia.

How normal is it to use these colours to describe anger? An English-speaker wishing to translate these examples might (erroneously) consider all three to be innovative, variations to the canonical form rosso di rabbia (red with rage). This preconception derives from the translator's L1 in which red with anger is the canonical expression; and while viola may not seem unusual because purple with rage is quite common (see Table 2 for frequencies), nero would appear odd as black is very uncommon, and in fact does not appear at all with either anger or rage in the Bank of English data.

Table 2. Colours of rage and anger in Italian and English.

\begin{tabular}{|l|l|}
\hline Italian & English \\
\hline nero di rabbia (8) & black (0) \\
rosso di rabbia (5) & red with anger (18)/ rage (28) \\
verde di rabbia (5) & green with anger (1)/ rage (2) \\
bianco di rabbia (2) & white with anger (7)/ rage (9) \\
blu dalla rabbia (1) & blue (0) \\
viola (0) & purple with anger (1)/ rage \\
rosa (0) & $(21)$ \\
& pink with anger (3)/ rage (2) \\
\hline
\end{tabular}

If these preconceptions are checked against Italian norms, as presented in the general reference corpus, a different picture emerges. The fact of the matter is that nero ( 8 of the 22 occurrences) is the most commonly used colour word in this context, followed by rosso and verde ( 5 each), and bianco (white). It therefore becomes apparent that the only unusual colour of the three that appear in the examples is viola, with both nero and verde being at least as frequent as the expected rosso.[10]

What are the implications of this for translation? If the corpus data shows that nero is commonly used in this pattern but black is not, how should the translator proceed? At this stage the principles of delexicalisation in conventionalised phraseology come back to centre stage. Nero di rabbia is unmarked, and the term which is correspondingly unmarked in English is red with anger/rage. By matching these expressions, the salient meaning of the colour word has to be ignored in favour of the unmarked phraseological meaning, which in this case is equivalent. The same is true for verde di rabbia, again an unmarked form. Should there be text-internal reasons for considering the colour to be relevant, the translator could use the alternative, livid; but if there are no special circumstances to take into consideration, then again red would serve to translate verde. The anomalous viola di rabbia would be inaccurately rendered by an unmarked form such as purple with rage, so some alternative rendering would be desirable; the most likely course of action would be to move away from the basic colour terms (Berlin \& Kay 1969) and select a particular shade such as plum, puce or even regal purple. In doing so, the colour is perceived accurately, but the effect of the SL original is preserved because the phrase is not normalised. 
Creative use of language may well make up only a small proportion of the language that is translated every day, but it is important both culturally and linguistically for a translator to render it in an appropriate manner. If the innovative and marked can be compared to related, unmarked forms in the SL, then the translator's job is facilitated greatly. By considering conventional language as largely delexicalised, and innovative language as being a combination of a delexical support and a contextually relevant addition, it is possible to go about achieving the same effect in the TL by adhering to the same principles, essentially re-creating the TL text in the same way as the SL text was constructed. In order to do this, however, the translator must have access to a large quantity of data from which to identify language norms, and that data comes in the form of general reference corpora. Smaller corpora are simply inadequate when it comes to dealing with stretches of text, fixed and semi-fixed phrases, and less-frequently used words and expressions, though they serve a fundamental role in the identification of genre-related phenomena.

\section{Discussion}

The use of comparable general reference corpora as an aid to the translation process is often onesided. TL corpora are often used as a control to ensure that the translation produced sounds natural, but less use is made of corpora in assessing the naturalness of the SL original. While must be acknowledged that absolute equivalence remains an elusive and rare phenomenon, adopting a dataassisted approach facilitates the identification of patterns and preferences across languages, making it possible to match up SL and TL expressions that are functionally as well as formally similar.

Choice in translation is related to choice in the SL, and this can be identified by comparing a given expression against its possible alternatives along the paradigmatic axis. In this way the translator obtains a more instant and detailed impression of the meaning being conveyed, and if an equivalent paradigm of choice is set up for the TL, the most suitable correspondences can be identified and used in the translated text.

Delexicalisation is fundamentally important as a concept when translating both conventional and unconventional language. There is a distinct difference between the meaning values of words in conventionalized utterances and their values in non-standard uses of the language, and this should be acknowledged and acted upon when translating text. Corpus data highlights the conventional and recurrent, and the rarity of unusual structures stands out. An awareness of the norms underlying non-standard and unconventional language makes it possible for the translator to recreate its effect in a structured and systematic way, rather than rely on the "intuition and hunch, inspiration and even flashes of genius" (Firth 1968: 85) that seem integral to the translation process.

Corpus tools are becoming increasingly sophisticated, and word profiling is therefore a much more straightforward matter than it was a few years ago. By teaching trainee translators how and when to use these tools their sensitivity to language patternings will be heightened, and their translations will improve in accuracy and fluency. A combination of automatic processing, manual analysis and greater awareness of how languages make meaning, will give translators the chance to have equivalence at their fingertips.

\section{Notes}

1. Although representativeness remains a moot point in corpus linguistics, it is beyond the scope of this paper to enter into the details of the argument: the reader is referred to Biber (1993) for a comprehensive account, and to Laviosa (1997) for considerations specific to comparable corpora.

2. See http://www.natcorp.ox.ac.uk/corpus/creating.xml for details of the text composition of the BNC. 
3. The author expresses her gratitude to the University of Birmingham/HarperCollins publishers for access to the full Bank of English for the duration of her PhD research, 1997-2003.

4. Access to CORIS is available by request http://corpora.dslo.unibo.it/ .

5. Since the time this research was undertaken, the substantially larger Italian corpus composed of texts from the Repubblica newspaper http://dev.sslmit.unibo.it/index.php has become available to researchers (see Aston \& Piccioni 2004). This corpus, taken in combination with CORIS, would constitute a general reference corpus for Italian comparable with the written component of the Bank of English in both size and content.

6. In particular, the reader is referred to the schematic representation of sorrow and its translation into German (Váradi and Kiss 2001: 169).

7. This represents the full paradigm of translations found in Ragazzini 1995. It should be noted that this is not an exhaustive list of every possible comparable expression, and it excludes paraphrasis.

8. The analysis of this data set made it evident that semantic prosodies cannot be identified for each node, but are specific to larger units of meaning which include the node and the particular collocational patternings which form around it: for this reason they are identified last of all.

9. One such application is Sketch Engine (Kilgarriff and Tugwell 2002, Kilgarriff et al. 2004) which runs on a variety of corpora in different languages http://www.sketchengine.co.uk/.

11. The single example of viola di rabbia was located on the Internet; there were no occurrences in the CORIS data.

\section{References}

Aston, G. and Piccioni, L. 2004. "Un grande corpus di italiano giornalistico.” In Atti del convegno nazionale AitLA, G. Bernini, G. Ferrari and M. Pavesi (eds). Perugia: Guerra. Available from http://www.sslmit.unibo.it/ guy/aitla repubblica.htm (accessed 25 March 2008).

Baker, M. 1992. In Other Words: A Coursebook on Translation. London/New York: Routledge.

Berlin, B. and Kay, P. 1969. Basic Color Terms: Their Universality and Evolution. Berkeley: University of California Press.

Biber, D. 1993.“Representativeness in Corpus Design." Literary and Linguistic Computing 8 (4): 243-257.

Firth, J.R. 1968 “A Synopsis of Linguistic Theory, 1930-5.” In Selected papers of J.R. Firth 19521957, F.R. Palmer (ed.), 168-205. London/Harlow: Longmans.

Glucksberg, S. and B. Keysar (1993) How metaphors work. In Metaphor and Thought (2nd and revised edition), A. Ortony (ed.), 401-424 Cambridge: Cambridge University Press.

Halliday, M.A.K. 1992. "Language Theory and Translation Practice.” Rivista internazionale di tecnica della traduzione 0 (pilot issue): 15-25.

Kenny, D. 2001. Lexis and Creativity in Translation. A Corpus-based Study. Manchester: St. Jerome.

Kilgarriff, A. and Tugwell, D. 2002. "Sketching words.” In Lexicography and Natural Language Processing: A Festschrift in Honour of B. T. S. Atkins, Marie-Hélène Corréard (ed), 125-137. Göteborg: EURALEX.

Kilgarriff, A., Rychly, P., Smrz, P. and Tugwell, D. 2004. "The Sketch Engine.” In Proceedings of the Eleventh EURALEX International Congress, 105-116. Lorient: Université de BretagneSud.

Krishnamurthy, R. 2000. "Collocation: from silly ass to lexical sets.” In Words in Context: A Tribute to John Sinclair on his Retirement, C. Heffer and H. Sauntson (eds), 31-47. Birmingham: The University of Birmingham.

Bowker, M. Cronin, D. Kenny and J. Pearson (eds), 101-112. Manchester: St. Jerome.

Laviosa, Sara. 1997. "How Comparable Can 'Comparable Corpora' Be?” Target 9 (2): 289-319.

Louw, W.E. 2000. "Some implications of progressive delexicalisation and semantic prosodies for Hallidayan metaphorical modes of expression and Lakoffian 'Metaphors we Live By'." Privately-distributed version of "Progressive delexicalization and semantic prosodies as early empirical indicators of the death of metaphors". Paper read at the 11th Euro-International 
Systemic Functional Workshop: Metaphor in systemic functional perspectives, University of Gent (Belgium), 14-17 July 1999.

Moon, R. 1998. Fixed Expressions and Idioms in English: A Corpus-Based Approach. Oxford: Clarendon.

Niemeier, S. (1998) "Colourless green ideas metonymise furiously" Rockstocker Beträge zur Sprachwissenschaft 5, 119-146.

Philip. G. 2003. Collocation and Connotation: a corpus-based investigation of colour words in English and Italian. PhD thesis. The University of Birmingham, UK. Available from http://amsacta.cib.unibo.it/archive/00002266 (accessed 25 March 2008)

Ragazzini, G. (ed.) 1995. Il Ragazzini: dizionario inglese italiano - italiano inglese (3rd edition). Bologna: Zanichelli.

Rossini Favretti, R. (2000) "Progettazione e costruzione di un corpus di italiano scritto: CORIS/CODIS.” In Linguistica e informatica: corpora, multimedialità e percorsi di apprendimento, R. Rossini Favretti (ed.), 39-56. Rome: Bulzoni.

Siepmann, D. 2005. "Collocation, colligation and encoding dictionaries. Part 1: Lexicological aspects." International Journal of Lexicography 18 (4): 409-443.

Sinclair, J.M. 1991. Corpus, Concordance, Collocation. Oxford: OUP.

Sinclair, J.M. 1996. "The Search for Units of Meaning." TEXTUS 9 (1), 75-106.

Tognini Bonelli, E. 2001. Corpus Linguistics at Work. Amsterdam and Philadelphia: John Benjamins.

Tognini Bonelli, E. 2002. "Functionally complete units of meaning across English and Italian: Towards a corpus-driven approach.” In Lexis in Contrast, B. Altenberg and S. Granger (eds), 73-95. Amsterdam and Philadelphia: John Benjamins.

Váradi, T. and Kiss, G. 2001. "Equivalence and Non-equivalence in Parallel Corpora." International Journal of Corpus Linguistics 6 (special issue): 167-177. 